\title{
Reconstruction of 3-D Histology Images by Simultaneous Deformable Registration
}

\author{
Marco Feuerstein, Hauke Heibel, José Gardiazabal, \\ Nassir Navab, and Martin Groher \\ microDimensions and Computer Aided Medical Procedures (CAMP), \\ Technische Universität München, Germany
}

\begin{abstract}
The reconstruction of histology sections into a 3-D volume receives increased attention due to its various applications in modern medical image analysis. To guarantee a geometrically coherent reconstruction, we propose a new way to register histological sections simultaneously to previously acquired reference images and to neighboring slices in the stack. To this end, we formulate two potential functions and associate them to the same Markov random field through which we can efficiently find an optimal solution. Due to our simultaneous formulation and the absence of any segmentation step during the reconstruction we can dramatically reduce error propagation effects. This is illustrated by experiments on carefully created synthetic as well as real data sets.
\end{abstract}

\section{Introduction}

Today, histology is still the gold standard for assessing anatomical information on a cellular level. Tissue samples are cut into ultra thin slices, stained, and viewed under a microscope. While traditional histology involves only a few slices to be analyzed, there is an increasing need to reassemble consecutive slices into a 3-D volume. Given such a volume, novel high-resolution in-vivo imaging techniques (e.g. micro-CT, high-field MRI, or phase contrast X-ray CT) can be validated, atlases can be created on a micron level, or 3-D micro-structures can be quantified for analysis. However, the geometrically coherent creation of such a 3-D histological volume is difficult to achieve, since the histological sectioning process introduces artifacts and distortions like holes, foldings, and tears.

There are currently two major approaches to create 3-D histological volumes: registration between consecutive sections [12910411 and registration of sections to external reference images coming from e.g. 3-D in-vivo imaging or 2-D block-face images acquired during histological sectioning [265]. A comprehensive overview of recent techniques is given by Cifor et al. [4]. When registering solely consecutive sections, the reconstructed structures are homogeneous but the aperture problem leads to drifts in the stack direction. These drifts cancel out real changes between neighboring sections, in particular for curved anatomical structures. This can be avoided by external reference images, but usually those images feature a smaller resolution or contrast than their corresponding 


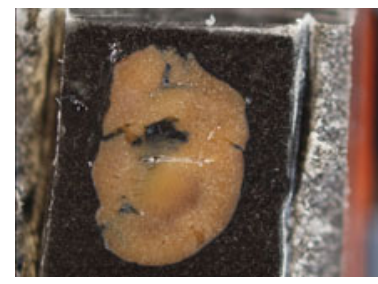

(a)

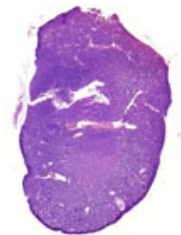

(b)

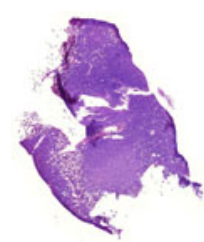

(c)

Fig. 1. Sample block-face image (a) its corresponding histology section (b), and a histology section that was heavily disrupted during cutting (c)

histological sections. Furthermore, the structural homogeneity between consecutive sections can hardly be guaranteed when aligning histological to reference images slice-by-slice.

To benefit from the advantages of both approaches, their sequential utilization has been proposed 133 . This can result in more homogeneous histological volumes of higher resolution, but comes at the cost of multiple sequential processing steps and may require a large number of empirically determined parameters. Moreover, the nature of sequential processing can easily annihilate improvements of a previous step and again worsen the overall structural homogeneity or continuity.

Our approach tackles this problem by registering histological sections simultaneously to their corresponding reference images and to their neighboring sections. We explicitly avoid any segmentation step during the registration process to keep propagated errors low. In order to efficiently solve the simultaneous alignment problem, we employ discrete optimization techniques for dense deformable registration using Markov random fields (MRFs) [7. In our MRF, unary potentials account for the registration to reference images, and two distinct pairwise potentials account for the registration to neighboring slices and for regularizing transformations, respectively. This model allows us to jointly register all sections to their respective reference while maintaining structural homogeneity. To the best of our knowledge, this is the first time where histology re-stacking is performed in a single process and solved efficiently by intensity-based registration using discrete optimization.

\section{Method}

Given a set of histology images $\mathcal{I}=\left\{\mathcal{I}_{1}, \ldots, \mathcal{I}_{n}\right\}$ and their corresponding blockface images $\mathcal{J}=\left\{\mathcal{J}_{1}, \ldots, \mathcal{J}_{n}\right\}$ (cf. Fig. 1a, b) we seek a set of sufficiently smooth transformations $\mathbf{T}=\left\{T_{1}, \ldots, T_{n}\right\}$, which align each $\mathcal{I}_{i}$ to $\mathcal{J}_{i}$ and to its adjacent neighbors $\mathcal{I}_{i-1}, \mathcal{I}_{i+1}$. This can be modeled by an energy minimization as

$$
\mathbf{T}^{*}=\arg \min _{\mathbf{T}} \mathbf{E}_{R}(\mathcal{I}, \mathcal{J}, \mathbf{T})+\mathbf{E}_{C}(\mathcal{I}, \mathbf{T})+\mathbf{E}_{S}(\mathbf{T}),
$$


where $\mathbf{E}_{R}(\mathcal{I}, \mathcal{J}, \mathbf{T})=\sum_{i=1}^{n} E_{R}\left(\mathcal{I}_{i} \circ T_{i}, \mathcal{J}_{i}\right)$ computes the energy between histology images and block-face images, $\mathbf{E}_{C}(\mathcal{I}, \mathbf{T})=\sum_{i=0}^{n-1} E_{C}\left(\mathcal{I}_{i} \circ T_{i}, \mathcal{I}_{i+1} \circ T_{i+1}\right)$ computes the energy between consecutive histology slices, and $\mathbf{E}_{S}(\mathbf{T})=\sum_{i=1}^{n} E_{S}\left(T_{i}\right)$ acts as an independent in-plane regularizer on each transformation $T_{i}$.

In the remainder of this section we will first explain the steps taken to prealign the given image stacks. Subsequently, we will focus on how to solve the optimization problem in a discrete framework.

\subsection{Rigid Pre-alignment}

To initialize and speed up our discrete optimization framework, we first rigidly align all pairs of histological sections and their corresponding block-face images. This can be fully automated by extracting and aligning the 2-D contours of our sample for each image pair, followed by a rigid registration. As our tissue sample is embedded in black paraffin as in 3 and hence clearly distinguishable from its background in both the histological and block-face images, we can use Otsu's automatic thresholding method to obtain all 2-D contours. For each pair of contours we compute their semi-major axes and centers based on moments and align them to obtain an initial rotation and translation for rigid registration. To be robust against the variability of intensities and visible structures between block-face and histological images, our rigid registration uses normalized mutual information.

\subsection{MRF Formulation of the Deformable Stack Registration}

We will now explain our MRF model for optimizing Eq. (11). In order to build an MRF model we first parameterize the transformations $\mathbf{T}$. We use a set of uniform free-form deformation (FFD) grids, i.e. the displacement field representing each $T_{i}$ is parameterized using 2-D FFDs based on cubic B-splines. Please note that we choose a stack of 2D FFD grids to model the independent transformations that happen while cutting each slice individually. The MRF is then constructed by assigning a node to each control point $\mathbf{p}^{i}$ of an FFD grid $\mathcal{G}^{i}$. We create two types of links between nodes: (a) between neighboring in-plane control points, which are located in the same FFD grid $\mathcal{G}^{i}$, and (b) between neighbors in consecutive FFD grids $\mathcal{G}^{i}, \mathcal{G}^{i+1}$, see Fig. 2.

We define a labeling $\mathbf{l}$ as the assignment of discrete values to nodes. We associate each label assignment $l_{\mathbf{p}}$ to a corresponding displacement $\mathbf{d}_{l_{\mathbf{p}}}$ of control point $\mathbf{p}$. For a given labeling $\mathbf{l}$ we can then cast $\mathbf{E}_{R}$ as a sum of unary terms, and $\mathbf{E}_{C}, \mathbf{E}_{S}$ as sums of pairwise terms, respectively, leading to an overall MRF energy $\mathbf{E}(\mathbf{l})$ :

$\mathbf{E}(\mathbf{l})=\sum_{i=1}^{n} \sum_{\mathbf{p} \in \mathcal{G}^{i}} \Theta_{R}^{i}\left(l_{\mathbf{p}}\right)+\gamma \sum_{i=1}^{n-1} \sum_{\substack{\mathbf{p} \in \mathcal{G}^{i} \\ \mathbf{q} \in \mathcal{G}^{i+1}}} \Theta_{C}^{i, i+1}\left(l_{\mathbf{p}}, l_{\mathbf{q}}\right)+\lambda \sum_{i=1}^{n} \sum_{\mathbf{p} \in \mathcal{G}^{i}} \sum_{\mathbf{r} \in \mathcal{N}(\mathbf{p})} \Theta_{S}^{i}\left(l_{\mathbf{p}}, l_{\mathbf{r}}\right)$, 


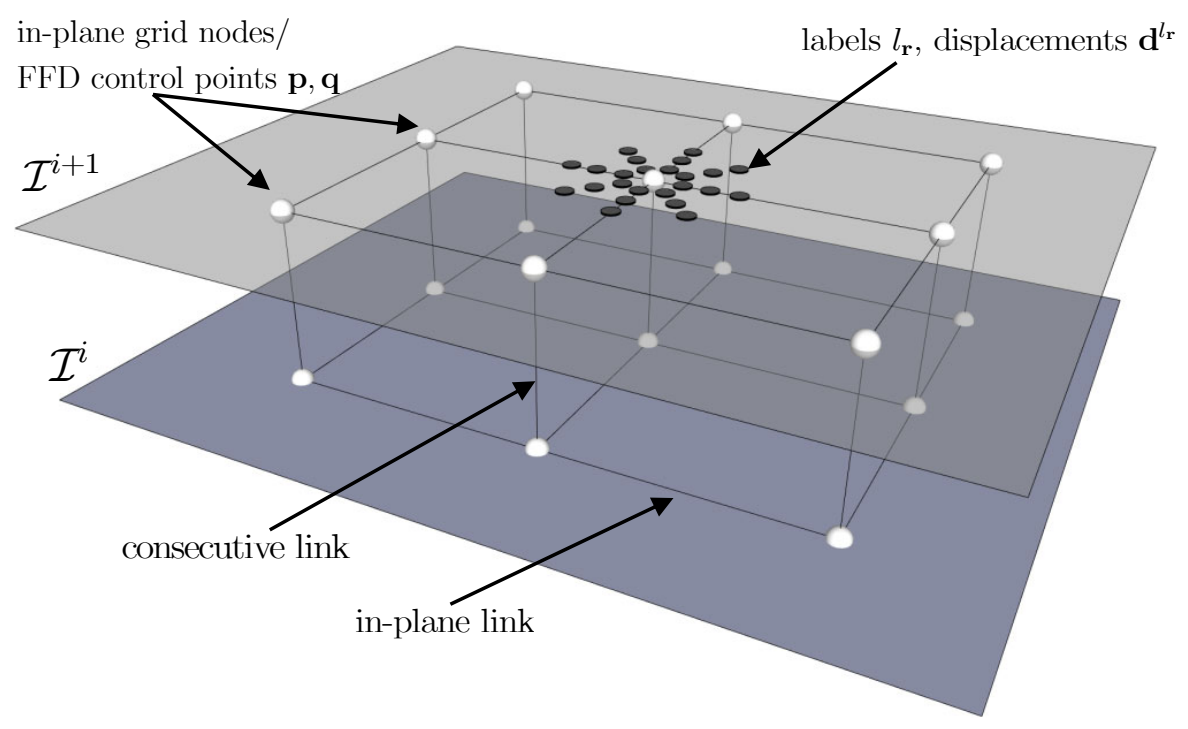

Fig. 2. MRF for two section images

where $\gamma$ and $\lambda$ are weighting factors to relate the three terms and $\mathcal{N}(\mathbf{p})$ denotes the set of in-plane neighbors of control point $\mathbf{p}$.

We can now compute costs for each term:

$$
\begin{aligned}
\Theta_{R}^{i}\left(l_{\mathbf{p}}\right) & \left.=\int_{\Omega_{i}} \eta(\mathbf{x}, \mathbf{p}) D_{1}\left(\mathcal{I}_{i}\left(\mathbf{x}+\mathbf{d}_{l_{\mathbf{p}}}\right)\right), \mathcal{J}_{i}(\mathbf{x})\right) d \mathbf{x} \\
\Theta_{C}^{i, i+1}\left(l_{\mathbf{p}}, l_{\mathbf{q}}\right) & =\int_{\Omega_{i}} \eta(\mathbf{x}, \mathbf{p}) \eta(\mathbf{x}, \mathbf{q}) D_{2}\left(\mathcal{I}_{i}\left(\mathbf{x}+\mathbf{d}_{l_{\mathbf{p}}}\right), \mathcal{I}_{i+1}\left(\mathbf{x}+\mathbf{d}_{l_{\mathbf{q}}}\right)\right) d \mathbf{x} \\
\Theta_{S}^{i}\left(l_{\mathbf{p}}, l_{\mathbf{r}}\right) & =\int_{\Omega_{i}} R\left(\mathbf{d}_{l_{\mathbf{p}}}, \mathbf{d}_{l_{\mathbf{r}}}\right) d \mathbf{x},
\end{aligned}
$$

where $D_{1}(.,),. D_{2}(.,$.$) compute intensity-based dissimilarity measures and R(.,$. computes a regularizing penalty to achieve in-plane smoothness of transformations. $\eta(\mathbf{x}, \mathbf{p})$ is a weighting factor, which controls the influence of control point $\mathbf{p}$ to pixel $\mathbf{x}$.

Given the discrete energy formulation $\mathbf{E}$, we can perform the simultaneous deformable registration using two specific dissimilarity measures $D_{1}, D_{2}$, a regularizer $R$, and a discrete optimization algorithm. In our particular application, we use normalized mutual information for $D_{1}$ and normalized cross correlation for $D_{2}$ assuming only linear intensity changes between consecutive slices. We choose to penalize the squared difference between neighboring displacement vectors, i.e. $R\left(\mathbf{d}_{l_{\mathbf{p}}}, \mathbf{d}_{l_{\mathbf{r}}}\right)=\left\|\mathbf{d}_{l_{\mathbf{p}}}-\mathbf{d}_{l_{\mathbf{r}}}\right\|^{2}$. For solving the discrete labeling problem (involving unary and pairwise terms only) we use the iterative quadratic pseudo-boolean optimization (QPBO) algorithm [8]. 


\section{Results}

We evaluated our method on synthetic data as well as histological sections of a rat kidney. To demonstrate the effect of our data term, we compare our method to a sole histology-to-block-face registration utilizing normalized mutual information, a registration only using consecutive histological sections utilizing normalized cross correlation, and a sequential, but not simultaneous combination of the two.

During deformable registration, we set most required parameters to the default values proposed in [7, i.e. the maximum allowed displacement of each level of the multi-scale approach is bound to the grid resolution, the sampling rate from the zero-displacement to the maximum displacement is 5 , sparse sampling is used, and 5 optimization cycles are performed on each pyramid level. We however use two image and control point resolution levels. Our label set scaling factor is set to $2 / 3$. We use 12 bins for the histograms needed for the computation of mutual information. Our weighting factors $\gamma$ and $\lambda$ are set to 0.2 and 2, respectively. These factors are determined experimentally and altering them did not vastly change the reconstruction results.

\subsection{Synthetic Data}

Our synthetic ground truth data resembles an ellipsoidal tissue sample embedded with skew tubular structures of varying diameter. To simulate the histological cutting process, we arbitrarily tear some of the sections and apply random FFDs to each section.

For realistic tearing we model each tear by its center, direction (pointing towards the closest point on the contour of the sectioned sample), and its apex angle. A tear symmetrically opens the sample within the apex angle and describes an elliptic sector whose pixels are all nullified. It also linearly affects its neighboring pixels within a specified influence angle, the further away from the direction vector, the less influence a pixel gets. The corresponding deformation field is stored as backward warping and has its maxima along the two vectors defining the elliptic sector. Deformations are linearly decreasing towards the direction vector as well as towards the vectors defined by the influence angle. Figure 3a shows a teared and deformed section.

We model staining and inherent intensity variabilities between block-face and histological images by inverting all original voxel intensities. We also add Gaussian noise to the ground-truth images (三block-face images) and to the deformed inverted images (三histology sections) to imitate the image acquisition process. Eventually we store the 2-D ground truth deformation fields for all pairs of deformed and ground truth images and run our method.

In order to quantitatively evaluate our method, we apply the performance measures commonly used for optical flow evaluation by the vision community 11. In detail, we compute the absolute endpoint error (EE) and the relative angular error (AE) between the ground truth and resulting deformation fields as well as the interpolation error (IE) and normalized IE (NE) between the ground truth and deformed images. Table 1 shows the measured errors for all compared 

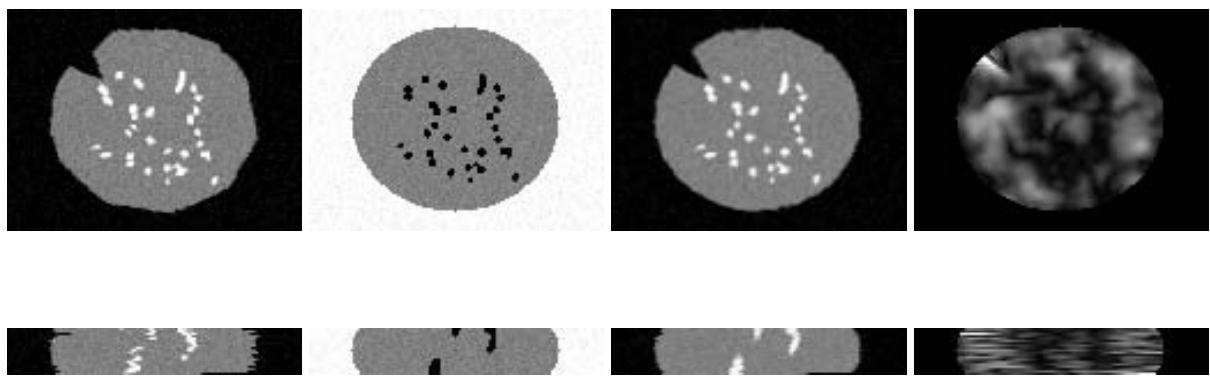

(a) source

(b) reference (c) result

(d) endpoint error

Fig. 3. Synthetic experimental data showing one section (top) and a cut through the

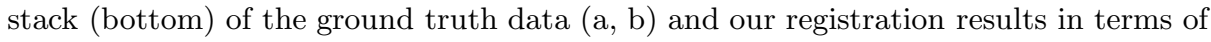
the deformed image (ㄷ) and its corresponding endpoint error (d)

methods, where relative improvements compared to the initial situation (where sections are assumed to be rigidly aligned) are included. Fig. [3d shows the EE of our simultaneous approach.

It can be seen that our proposed method achieves the largest relative improvement of each error measure. The registration only comprising consecutive sections performs worst - this is due to a drift of the stack images, which increases errors in the deformation fields (AE, EE) and hence differences between the ground truth reference and deformed images (IE, NE). Moreover, it should be noted that a sequential application of histology-to-block-face and consecutive registration can decrease the quality of the alignment: the results are worse than a registration based on block-face images only, which is again related to the global drift mentioned above.

\subsection{Rat Kidney}

The rat kidney sample was cut into $9 \mu \mathrm{m}$ thin sections (see Fig. 1b for a sample image). Before each cut we acquired a block-face image (cf. Fig. 1a) using an Olympus E-620 SLR camera with a $50 \mathrm{~mm} \mathrm{1:2} \mathrm{macro} \mathrm{objective.} \mathrm{About} \mathrm{5 \%} \mathrm{of}$ our sections were disrupted during cutting, which is usually unavoidable in the histological sectioning process (cf. Fig. 1c for an example). After staining all sections with hematoxylin and eosin, we digitized them using a MIRAX MIDI whole slide scanner by Carl Zeiss. Using a grid spacing of $1 \mathrm{~mm}$, we exemplarily run our registration algorithm on a stack of 580 sections (including the disrupted sections) of $1008 \times 756$ pixels and a pixel spacing of $33.38 \mu \mathrm{m}$, which took about 2.5 hours to complete on a workstation with 24 GB memory and 8 cores.

Figure 4 shows a cut in the middle of the volume orthogonal to slicing direction. Out of all techniques, ours produces the most similar structures compared to the uncut situation. In the close-ups, it can be observed that our method (Fig. 4h) performs satisfactory even in the presence of disrupted slices, while the consecutive (Fig. 4f) and sequential (Fig. 4g) methods fail and histology-toblock-face registration (Fig. 4e) produces large jitter. 
Table 1. Experiments on synthetic data. The values in brackets are the relative improvements compared to the initial alignment.

\begin{tabular}{ccccc}
\hline & \multicolumn{4}{c}{ error measure } \\
\cline { 2 - 5 } method & $\mathrm{AE}$ & $\mathrm{EE}$ & $\mathrm{IE}$ & $\mathrm{NE}$ \\
\hline initial alignment & 50.84 & 1.57 & 26.06 & 13.28 \\
\hline histology-to-block-face & 36.26 & 1.22 & 17.70 & 9.13 \\
registration & $(+28.68 \%)$ & $(+22.29 \%)$ & $(+32.08 \%)$ & $(+31.25 \%)$ \\
consecutive registration & 57.41 & 1.91 & 25.76 & 12.35 \\
& $(-12.92 \%)$ & $(-21.66 \%)$ & $(+1.15 \%)$ & $(+7.00 \%)$ \\
sequential approach & 36.56 & 1.23 & 24.97 & 11.72 \\
& $(+28.09 \%)$ & $(+21.66 \%)$ & $(+4.18 \%)$ & $(+11.75 \%)$ \\
simultaneous approach & $\mathbf{3 4 . 3 0}$ & $\mathbf{1 . 1 7}$ & $\mathbf{1 6 . 6 9}$ & $\mathbf{8 . 6 6}$ \\
& $(+\mathbf{3 2 . 5 3 \%})$ & $(+\mathbf{2 5 . 4 8 \%})$ & $\mathbf{( + 3 5 . 9 6 \% )}$ & $(+\mathbf{3 4 . 7 9 \%})$ \\
\hline
\end{tabular}

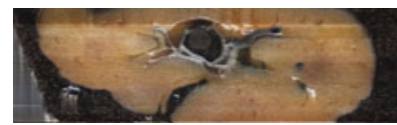

(a) block-face (bf)

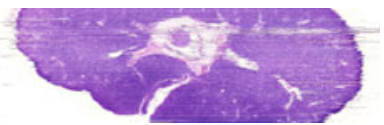

(b) rigid pre-alignment

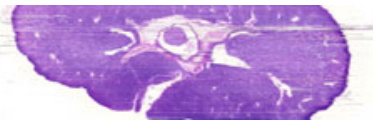

(c) simultaneous

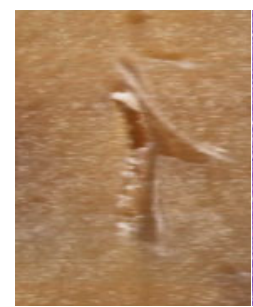

(d) block-face

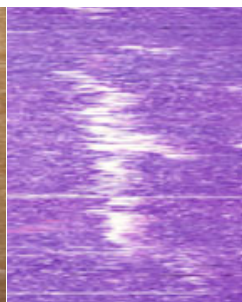

(e) histo-to-bf

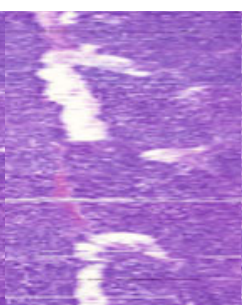

(f) consecutive

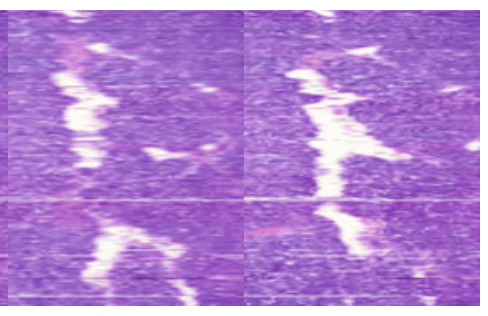

(g) sequential (h) simultaneous

Fig. 4. First row: sagittal cuts through the kidney. The first image is generated from block-face images, middle and right show histology images before and after simultaneous registration. Second row: exemplary close-up of a vessel structure.

\section{Conclusion}

In this paper we develop a method for the fully automatic reconstruction of 3-D histology stacks. Our approach guarantees geometrical coherence by combining the registration of sections to block-face images with a registration between neighboring slices. This allows us to undo deformations induced by cutting while aligning anatomical structures that are not apparent in the block-face images. Experimental results show that our simultaneous registration algorithm is not only accurate, but also robust against artifacts produced during the cutting process. Moreover, its sensitivity to misalignment due to grossly corrupted slices 
is almost negligible. Motivated by our excellent results, we intend to extend our current implementation with adequate streaming techniques to be able to increase the resolution of input and output data in the future.

Acknowledgments. We want to thank F. Pfeiffer and M. Bech from the physics department (E17), Technische Universität München, for providing the kidney data set. Parts of this research were supported by the Alexander von Humboldt Foundation providing a Feodor Lynen Research Fellowship and the Excellence Cluster Initiative, Munich Center for Advanced Photonics.

\section{References}

1. Baker, S., Scharstein, D., Lewis, J., Roth, S., Black, M.J., Szeliski, R.: A database and evaluation methodology for optical flow. IJCV 92(1), 1-31 (2011)

2. Bardinet, E., Ourselin, S., Dormont, D., Malandain, G., Tandé, D., Parain, K., Ayache, N., Yelnik, J.: Co-registration of histological, optical and MR data of the human brain. In: Dohi, T., Kikinis, R. (eds.) MICCAI 2002. LNCS, vol. 2488, pp. 548-555. Springer, Heidelberg (2002)

3. Chakravarty, M.M., Bedell, B.J., Zehntner, S.P., Evans, A.C., Collins, D.L.: Threedimensional reconstruction of serial histological mouse brain sections. In: ISBI, pp. 987-990 (2008)

4. Cifor, A., Pridmore, T., Pitiot, A.: Smooth 3-D reconstruction for 2-D histological images. In: Prince, J.L., Pham, D.L., Myers, K.J. (eds.) IPMI 2009. LNCS, vol. 5636, pp. 350-361. Springer, Heidelberg (2009)

5. Dauguet, J., Delzescaux, T., Cond, F., Mangin, J., Ayache, N., Hantraye, P., Frouin, V.: Three-dimensional reconstruction of stained histological slices and 3D non-linear registration with in-vivo mri for whole baboon brain. Journal of Neuroscience Methods 164, 191-204 (2007)

6. Gefen, S., Tretiak, O., Nissanov, J.: Elastic 3D alignment of rat brain histological images. IEEE TMI 22(11), 1480-1489 (2003)

7. Glocker, B., Komodakis, N., Tziritas, G., Navab, N., Paragios, N.: Dense image registration through MRFs and efficient linear programming. MedIA 12(6), 731741 (2008)

8. Kolmogorov, V., Rother, C.: Minimizing nonsubmodular functions with graph cutsa review. IEEE PAMI 29(7), 1274-1279 (2007)

9. Schmitt, O., Modersitzki, J., Heldmann, S., Wirtz, S., Fischer, B.: Image registration of sectioned brains. IJCV 73(1), 5-39 (2006)

10. Tan, Y., Hua, J., Dong, M.: Feature curve-guided volume reconstruction from 2D images. In: ISBI, pp. 716-719 (April 2007)

11. Bagci, U., Bai, L.: Automatic best reference slice (BRS) selection for smooth volume reconstruction of a mouse brain from histological images. IEEE TMI 29(9), 1688-1696 (2010)

12. Wirtz, S., Papenberg, N., Fischer, B., Schmitt, O.: Robust and staining-invariant elastic registration of a series of images from histologic slices. In: Medical Imaging: Image Processing, vol. 5747, pp. 1256-1262 (2005)

13. Yushkevich, P.A., Avants, B.B., Ng, L., Hawrylycz, M., Burstein, P.D., Zhang, H., Gee, J.C.: 3D mouse brain reconstruction from histology using a coarse-to-fine approach. In: Pluim, J.P.W., Likar, B., Gerritsen, F.A. (eds.) WBIR 2006. LNCS, vol. 4057, pp. 230-237. Springer, Heidelberg (2006) 